\title{
Interleukin 17 receptor E identifies heterogeneous T helper 17 cells in peritoneal fluid of severe endometriosis patients
}

\author{
Yanping Jiang ${ }^{1}$, Lu Wang ${ }^{1}$, Yaqin Peng ${ }^{1}$, Jian Qin ${ }^{1}$, Aili Tan ${ }^{1}$, and Shujun Wang ${ }^{1}$ \\ ${ }^{1}$ Renmin Hospital of Wuhan University
}

July 27, 2021

\begin{abstract}
Endometriosis is a chronic inflammatory disorder resulting in pelvic pain and infertility. The role of T helper 17 (Th17) cells in endometriosis remains elusive. In this study, through detecting CXCR3, CCR4, CCR10, CCR6, interleukin-17 Receptor E (IL-17RE), and CD27, live ROR rt-and-IL-17A-expressing Th17 cells were distinguished and sorted from peritoneal fluid (PF) of patients with stage III and IV endometriosis. Furthermore, we found that IL-17RE and CD27 were the labels of heterogeneous PF Th17 subsets, i.e. IL-17RE-CD27- subset, IL-17RE+CD27- subset, and IL-17RE+CD27+ subset. The former two subsets expressed higher IL-17A, GM-CSF, and IL-22 and were more proliferative than the latter subset. RNA-Seq analysis on IL17RE+ Th17 subset and IL-17RE- Th17 subset revealed up-regulation of genes involved in oxidative phosphorylation and electron transport chain in IL-17RE+ Th17 subset relative to IL-17RE- Th17 subset. Consistently, the IL-17RE+ Th17 subset produced more adenosine triphosphate (ATP) and reactive oxygen species (ROS) than IL-17RE- Th17 subset. In conclusion, this study provides a novel method to detect and isolate live PF Th17 cells from endometriosis patients and unveils the functional and metabolic heterogeneity of PF Th17 subsets. Therefore, it sheds light on the elucidation of molecular mechanisms that modulate the function of pathological Th17 cells in endometriosis.
\end{abstract}

Interleukin 17 receptor $E$ identifies heterogeneous $T$ helper 17 cells in peritoneal fluid of severe endometriosis patients

Yanping Jiang ${ }^{1}$, Lu Wang ${ }^{* 1}$,Yaqin Peng ${ }^{1}$, Jian Qin ${ }^{2}$, Aili Tan ${ }^{1}$, Shujun Wang ${ }^{1}$

${ }^{*}$ Co-first authors

1. Department of Gynaecology, Renmin Hospital of Wuhan University, Wuhan, Hubei 430060

2. Renmin Hospital of Wuhan University, Wuhan, Hubei 430060

Correspondence to:Professor Yanping Jiang, Department of Gynaecology, Renmin Hospital of Wuhan University, 238 Jiefang Road, Wuhan, Hubei 430060, P.R. China E-mail: jiang_yanping@whu.edu.cn

Running title: IL-17RE marks Th17 heterogeneity in endometriosis

Abstract: Endometriosis is a chronic inflammatory disorder resulting in pelvic pain and infertility. The role of T helper 17 (Th17) cells in endometriosis remains elusive. In this study, through detecting CXCR3, CCR4, CCR10, CCR6, interleukin-17 Receptor E (IL-17RE), and CD27, live ROR $\gamma$ t-and-IL-17A-expressing Th17 cells were distinguished and sorted from peritoneal fluid (PF) of patients with stage III and IV endometriosis. Furthermore, we found that IL-17RE and CD27 were the labels of heterogeneous PF Th17 subsets, i.e. IL-17RE-CD27- subset, IL-17RE+CD27- subset, and IL-17RE+CD27+ subset. The former two subsets expressed higher IL-17A, GM-CSF, and IL-22 and were more proliferative than the latter subset. RNA-Seq analysis on IL-17RE+ Th17 subset and IL-17RE- Th17 subset revealed up-regulation of genes involved in oxidative phosphorylation and electron transport chain in IL-17RE+ Th17 subset relative to IL-17RE- Th17 
subset. Consistently, the IL-17RE+ Th17 subset produced more adenosine triphosphate (ATP) and reactive oxygen species (ROS) than IL-17RE- Th17 subset. In conclusion, this study provides a novel method to detect and isolate live PF Th17 cells from endometriosis patients and unveils the functional and metabolic heterogeneity of PF Th17 subsets. Therefore, it sheds light on the elucidation of molecular mechanisms that modulate the function of pathological Th17 cells in endometriosis.

Keywords:Endometriosis, T helper 17 (Th17) cells, Interleukin 17 Receptor E, CD27, Oxidative phosphorylation, Electron transport chain.

\section{Introduction}

Featuring the presence of endometrial tissue outside the uterine cavity, endometriosis is a chronic inflammatory disorder resulting in pelvic pain and infertility [1]. Various immune cell types including neutrophils, macrophages, natural killer (NK) cells, dendritic cells, and T lymphocytes play significant roles in the initiation and progression of endometriosis [2]. Immune cells secret biochemical factors that facilitate endometriotic cell division, invasion, as well as angiogenesis [3]. However, the exact functions and regulatory mechanisms of these immune cells have not been thoroughly understood.

Previous findings suggest that imbalanced $\mathrm{T}$ cell subsets and/or $\mathrm{T}$ cell dysfunction significantly contribute to the elevation of pathological cytokines and inflammatory reactions at endometriosis tissues. Cytokines released by peritoneal $\mathrm{T}$ cells are predominantly $\mathrm{T}$ helper 2 (Th2) cytokines in endometriosis tissue [4]. Regulatory $\mathrm{T}$ cell deficiency seems to promote local inflammation, angiogenesis, and facilitate the attachment and growth of endometrial implants [5]. Recent studies have revealed the role of T helper 17 (Th17) cells in endometriosis pathophysiology. Th17 cells are defined by the secretion of IL-17A, a pro-inflammatory cytokine crucial for the development of inflammatory and autoimmune diseases. The proportion of Th17 cells in endometriosis lesions is higher than that in the normal endometrium [6], and their high proportion in peritoneal fluid (PF) is related to the severity of endometriosis [7]. Additionally, the functional features and underlying regulatory mechanisms of Th17 cells in endometriosis are yet to be completely understood. It is, therefore, important to isolate live Th17 cells from blood, lymphoid organs, PF, and endometriosis lesions to disclose the molecular characteristics critical to their activities. Unfortunately, previous and current Th17 studies in endometriosis patients rely on intracellular staining of IL-17A for flow cytometry analysis, making the isolation of live Th17 cells impractical. A novel approach to sort live Th17 cells is in urgent demand for endometriosis research. Several research groups have used an array of chemokine receptors to dissect $\mathrm{T}$ cell subsets including Th17 cells in other diseases [8-11]. However, whether these receptors apply to Th17 detection in endometriosis remains untested.

In the present study, we use a set of cell surface markers to detect and sort live Th17 cells from PF of patients with advanced/severe endometriosis. We found that CCR4, CCR6, IL-17RE, and CD27 defined live PF Th17 cells. Moreover, RNA-Seq analysis revealed significant distinct transcript profiles of PF Th17 subpopulations. Therefore, our data disclose the heterogeneity of PF Th17 cells in endometriosis.

\section{Materials and methods}

\section{Patients}

This study was approved by the Research Ethics Committee of Renmin Hospital of Wuhan University with written informed consent obtained from all participants. Between April 2018 and November 2020 at the Department of Gynaecology, Renmin Hospital of Wuhan University, 48 women with stage III-IV endometriosis according to the revised American Fertility Society score (r-AFS) (aged 28-35, mean age=30.3 years) were recruited for analysis.

\section{Enrichment of mononuclear cells from PF}

Under general anesthesia, PF was collected from the pouch of Douglas at the start of laparoscopy. Any blood-containing PF was discarded and not included in the study. PF mononuclear cells were overlaid on the lymphocyte separation medium (Sigma-Aldrich) and enriched by density-gradient centrifugation at 400 
rcf for 20 minutes. Cells in the interface were harvested, washed with PBS, and incubated in $200 \mu \mathrm{l}$ of red blood cell lysis buffer (ThermoFisher) for 15 minutes at room temperature to remove any residual red blood cells. The cells were then washed with PBS and suspended in PBS containing $2 \mathrm{mM}$ EDTA and $0.5 \%$ bovine serum albumin (BSA) for flow cytometry.

\section{Flow cytometry}

For Th17 detection and sorting, the following antibodies were used: APC/Cy7 anti-CD3 (HIT3a), FITC antiCD4 (A161A1), PE anti-CCR4 (L291H4), PE/Cy7 anti-CXCR3 (G025H7), PerCP/Cy5.5 anti-CCR6 (292L17), APC anti-CCR10 (6588-5), PE anti-CD14 (63D3), PE anti-CXCR3 (G025H7), PE anti-CCR10 (65885), PE/Cy7 anti-CCR4 (L291H4), APC/Cy7 anti-CD27 (M-T271), Pacific blue anti-IL-17A (BL168), and Pacific blue anti-GM-CSF (BVD2-21C11) were purchased from BioLegend. APC anti-IL-17RE (FAB8358A) was purchased from R\&D Systems. To stain cell surface markers, PF mononuclear cells were incubated with $2 \mu \mathrm{g} / \mathrm{ml}$ of each antibody for $30 \mathrm{~min}$ on ice before analysis and sorting on a BD FACSAria Flow Cytometer (BD biosciences). To stain intracellular cytokines after surface marker staining, cells were fixed with 200 $\mu \mathrm{l}$ of $2 \%$ paraformaldehyde for 20 minutes, followed by permeabilization in $1 \mathrm{ml}$ of $90 \%$ methanol-PBS on ice for $25 \mathrm{~min}$. Cells were then incubated with $5 \mu \mathrm{g} / \mathrm{ml}$ of each antibody for 1 hour at room temperature, respectively. Cells were washed and suspended in PBS for analysis on an LSRII flow cytometer with a UV laser (BD biosciences).

\section{RNA-Seq}

PF Th17 cell subpopulations were sorted from 5 patients and pooled for RNA-Seq. Cellular RNA was extracted using the Arcturus PicoPure RNA Isolation Kit (ThermoFisher). The quality and quantities of RNA samples were measured with the QIAxcel RNA QC Kit v2.0 (Qiagen) following the manufacturer's instructions. One microgram RNA at the concentration $>200 \mathrm{ng} / \mu \mathrm{l}$ was subjected to library construction, sequencing, reads quality check, alignment, clustering, expression assessment, Gene Ontology (GO) enrichment analysis, and Kyoto Encyclopedia of Genes and Genomes (KEGG) enrichment analysis by the Wuhan SeqHealth Co., Ltd. The Collibri ${ }^{\mathrm{TM}}$ Stranded RNA Library Prep Kit for Illumina (ThermoFisher), HiSeq 2000 Sequencing System (Illumina), TopHat v2.1.1, HTSeq v0.12.4, DEGseq2, and Hypergeometric test were used for the above procedures, respectively.

\section{Validation of DEGs by quantitative polymerase chain reaction (qPCR)}

cDNA preparation was achieved using the PrimeScript 1st strand cDNA Synthesis Kit (Takara Bio). qPCR was implemented using the SYBR Green Quantitative RT-PCR (Sigma-Aldrich) on an Azure Cielo 3 RealTime qPCR System (Azure Biosystems). The PCR cycle condition was $50^{\circ} \mathrm{C}$ for 2 minutes, $95^{\circ} \mathrm{C}$ for 10 minutes, $95^{\circ} \mathrm{C}$ for 15 seconds and $60^{\circ} \mathrm{C}$ for 30 seconds in 40 cycles, then $72^{\circ} \mathrm{C}$ for 10 minutes. After normalization to $\beta$-actin mRNA level, the relative transcript amounts were determined by the $2^{-\Delta \Delta^{\prime \prime} \tau}$ method. The primer sequences are displayed in Supplementary Table 1\&2 .

Table. 1 Twenty Up-regulated Metabolic Genes in IL-17RE ${ }^{+}$Th17 Cells Gene

NDUFB5

IDH1

$M T-C O 1$

UQCRC1

NDUFS6

SDHAF 4
Table. 1 Twenty Up-regulated Metabolic Genes in IL-17RE ${ }^{+}$Th17 Cells Protein

NADH dehydrogenase [ubiquinone] 1 beta subcomplex subunit 5 Isocitrate dehydrogenase [NADP +$]$ Cytoplasmic Cytochrome c oxidase subunit 1 Cytochrome b-c1 complex subunit 1 NADH dehydrogenase [ubiquinone] iron-sulfur protein 6

\section{Succinate dehydrogenase assembly factor 4}




$\begin{array}{ll}\text { ATPAF1 } & \begin{array}{l}\text { ATP synthase mitochondrial F1 complex assembly } \\ \text { factor } 1\end{array} \\ \text { CyDB } & \text { Cytochrome bd-I ubiquinol oxidase subunit } 2 \\ \text { Succinate dehydrogenase [ubiquinone] cytochrome } & \text { b small subunit } \\ \text { SDHD } & \text { Phosphoglycerate kinase } 1 \\ \text { PGK1 } & \text { Cytochrome b-c1 complex subunit } 7 \\ \text { UQCRB } & \text { NADH dehydrogenase [ubiquinone] } 1 \text { subunit } \mathrm{C} 2 \\ \text { NDUFC2 } & \text { NADH dehydrogenase [ubiquinone] } 1 \text { alpha } \\ \text { NDUFA5 } & \text { subcomplex subunit } 5 \\ \text { CyT-CO2 } & \text { NADH dehydrogenase [ubiquinone] complex I, } \\ \text { NDUFAF6 } & \text { assembly factor } 6 \\ & \text { ATP synthase mitochondrial F1 complex assembly } \\ \text { ATPAF1A } & \text { factor } 1 \\ & \text { NADH-ubiquinone oxidoreductase chain } 3 \\ M T-N D 3 & \text { Succinate dehydrogenase assembly factor } 4 \\ \text { SDH8 } & \text { Protoheme IX farnesyltransferase } \\ \text { COX10 } & \text { NADH dehydrogenase [ubiquinone] } 1 \text { alpha } \\ \text { NDUFA3 } & \text { subcomplex subunit } 3 \\ & \end{array}$

\begin{tabular}{|c|c|}
\hline $\begin{array}{l}\text { Table. } 2 \text { Twenty Down-regulated Metabolic Genes in IL-17RE }+ \text { Th17 Ce } \\
\text { Gene }\end{array}$ & $\begin{array}{l}\text { Table. } 2 \text { Twenty Down-regul } \\
\text { Protein }\end{array}$ \\
\hline$M T-N D 3$ & NADH-ubiquinone oxidoreducta \\
\hline$D L A T$ & Dihydrolipoyllysine-residue acet \\
\hline$M T-A T P 6$ & F-ATPase protein 6 \\
\hline IDHЗB & Isocitrate dehydrogenase [NAD] \\
\hline CYP51A1 & Lanosterol 14-alpha demethylase \\
\hline$H M G C S 1$ & Hydroxymethylglutaryl-CoA sy \\
\hline$A C A D L$ & Long-chain specific acyl-CoA de \\
\hline COQ5 & 2-methoxy-6-polyprenyl-1,4-benzo \\
\hline$G C D H$ & Glutaryl-CoA dehydrogenase \\
\hline TPI1 & Triosephosphate isomerase \\
\hline$L P C A T 4$ & Lysophospholipid acyltransferase \\
\hline PРAPथA & Lipid phosphate phosphohydrolase \\
\hline DHCR24 & $\operatorname{Delta}(24)$-sterol reductase \\
\hline$H M G C L$ & Hydroxymethylglutaryl-CoA lyase \\
\hline$A C A D M$ & Medium-chain specific acyl-CoA \\
\hline GLB1L & Beta-galactosidase-1-like protein \\
\hline ENPP6 & Glycerophosphocholine cholinepho \\
\hline$S G P L 1$ & Sphingosine-1-phosphate lyase 1 \\
\hline GAL3ST1 & Galactosylceramide sulfotransfe \\
\hline$B P G M$ & Bisphosphoglycerate mutase \\
\hline
\end{tabular}

\section{Cell cycle analysis}

Sorted Th17 subpopulations were fixed in ice-cold $70 \%$ ethanol for 2.5 hours. Cells were centrifuged for 5 min at $300 \mathrm{rcf}$ at $4{ }^{\circ} \mathrm{C}$. Ethanol was removed and cells were rinsed once with ice-cold Hank's balanced salt solution (HBSS) containing $\mathrm{Mg}^{2+}$ and $\mathrm{Ca}^{2+}$. After that, cells were suspended in $0.5 \mathrm{ml}$ of ice-cold HBSS containing $\mathrm{Mg}^{2+}$ and $\mathrm{Ca}^{2+}$. The cell suspension was mixed with an equal volume of HBSS containing $2 \mathrm{mg}$ 
Hoechst 33342 (Molecular Probes) and $4 \mathrm{mg}$ Pyronin Y (Sigma-Aldrich) and incubated for 20 minutes in the dark. Cells were then loaded on the flow cytometer for cell cycle analysis.

\section{Adenosine triphosphate (ATP) assay}

The ATP amount evaluation was conducted using the Luminescent ATP Detection Assay Kit (ab113849, Abcam) following the manufacturer's protocol.

\section{2, 7-dichlorodihydrofluorescein diacetate (H2DCFDA) staining}

Reactive oxygen species (ROS) in Th17 cells were assessed by H2DCFDA staining. Th17 cells were incubated in pre-warmed PBS containing $10 \mu \mathrm{M}$ H2DCFDA (ThermoFisher) for 15 minutes at $37^{\circ} \mathrm{C}$. After two washes with PBS, cells were suspended in PBS and the green fluorescence was measured on the flow cytometer.

\section{Statistics}

Each experiment was independently performed 2 or 3 times. The data were shown as mean \pm standard deviation unless specified. The Student's t-test, non-parametric Mann-Whitney U test or Kruskal-Wallis test was used to analyze the statistical significance. A $p$-value $<0.05$ was regarded as significant.

\section{Results}

\section{The phenotype of PF IL-17-producing T cells in endometriosis}

Several cell surface markers have been applied to distinguish $\mathrm{T}$ cell subsets $[12,13]$. To characterize the phenotype of PF Th17 cells, we first detected the expression of these markers on IL-17-producing T cells in PF of endometriosis patients. As shown in Figure 1A , a substantial PF CD3 ${ }^{+}$IL-17-producing T cell population was observed. Further detection revealed that these cells were $\mathrm{CD}^{+}{ }^{+} \mathrm{CXCR} 3^{-}$(Figure 1B) . Based on the expression of CCR 6 and CCR 4 , these cells can be divided into CCR $6^{+} \mathrm{CCR} 4^{+}$and CCR $6^{+} \mathrm{CCR} 4^{-}$ subpopulations, respectively (Figure 1B \& 1C). Based on the expression of IL-17RE and CD27, these cells can be divided into IL-17RE ${ }^{+}$CD27 $7^{-}$subset, IL-17RE ${ }^{+}$CD27 ${ }^{+}$subset, IL-17RE-CD27 ${ }^{+}$subset (very few or almost absent in some samples), and IL-17RE-CD27- subset (Figure 1B \& 1D) .

Because IL-17-producing T cells might involve both Th17 and Th1/17 cells, we needed to accurately discriminate these two populations to isolate live Th17 cells for further investigations. To this end, we analyzed PF CD $3{ }^{+} \mathrm{CD}^{+} \mathrm{T}$ cells(Figure 2A) for the expression of CCR6, CCR4, CXCR3, and CCR10. As shown in Figure 2B , within PF CD $3^{+} \mathrm{CD} 4^{+} \mathrm{T}$ cells, four subpopulations were seen based on the expression of CCR 4 and CXCR3: CCR4 ${ }^{+} \mathrm{CXCR}^{-}$cells, $\mathrm{CCR} 4^{+}{ }^{-} \mathrm{CXCR} 3^{+}$cells, $\mathrm{CCR} 4^{-} \mathrm{CXCR} 3^{+}$cells, and $\mathrm{CCR} 4{ }^{-} \mathrm{CXCR} 3^{-}$cells. We then sorted the four subpopulations and measured the expression of $\mathrm{T}$ cell subset master regulators, i.e. T-bet (Gene name TBX21), GATA3, and ROR rt. As shown in Figure 2C, T-bet was highly expressed in CCR4 ${ }^{-} \mathrm{CXCR}^{+}$cells and GATA3 was highly expressed in $\mathrm{CCR} 4^{+} \mathrm{CXCR} 3^{-}$cells, whereas ROR ${ }^{+}$t was also significantly CCR $4^{+} \mathrm{CXCR}^{-}$cells. This suggested that CCR $4^{+}{ }^{-} \mathrm{XCR} 3^{-}$cells contained both Th2 and Th17 cells. Interestingly, CCR4 ${ }^{-} \mathrm{CXCR} 3^{+} \mathrm{T}$ cells, which expressed high T-bet, also expressed moderate levels of ROR $r$ t, suggesting that this subpopulation harbored Th1 and Th1/17 cells.

We then further dissected CCR $4^{+}$CXCR $3^{-} \mathrm{T}$ cells based on the expression of CCR 6 and CCR 10 . As shown in Figure 2D , four subpopulations were observed: CCR $6^{+} \mathrm{CCR} 10^{-}$cells, CCR6 ${ }^{+} \mathrm{CCR} 10^{+}$cells, CCR6CCR $10^{+}$cells, and CCR6 $6^{-} \mathrm{CCR} 10^{-}$cells. We then sorted these cells to check the expression of T-bet, GATA3, and ROR rt. T-bet expression was ubiquitously low in all four subpopulations, while GATA3 was highly expressed in the CCR6 $6^{-} \mathrm{CCR} 10^{-}$subpopulation(Figure 2E). ROR $\gamma$ t was robustly expressed in the $\mathrm{CCR}^{+}{ }^{+} \mathrm{CCR} 10^{-}$subpopulation but low in the other three subpopulations (Figure 2E) . Therefore, Th17 cells were $\mathrm{CCR} 6^{+} \mathrm{CCR} 4^{+} \mathrm{CXCR} 3^{-} \mathrm{CCR} 10^{-}$.

To precisely study live PF Th17 cells, we needed to introduce more fluorophore-conjugated antibodies to label Th17 cells. However, it is difficult for conventional flow cytometers without ultraviolet lasers to recognize more than 6 fluorophores. Therefore, we came up with a new method to distinguish live Th17 cells. PEconjugated anti-CD14 antibody, anti-CXCR3 antibody, and anti-CCR10 antibody were used together to 
exclude $\mathrm{CD} 14^{+}$macrophages, CXCR3 ${ }^{+}$Th1 cells, CXCR3 ${ }^{+}$Th1/17 cells, and other non-Th2 and nonTh17 $\mathrm{T}$ cells (Figure 3A) . Within the CD14 ${ }^{-} \mathrm{CXCR} 3^{-} \mathrm{CCR} 10^{-}$cells, $\mathrm{CD} 4^{+} \mathrm{T}$ cells were divided into four subpopulations according to the expression of CCR6 and CCR4: $\mathrm{CCR} 6^{+} \mathrm{CCR} 4^{-}$cells, $\mathrm{CCR} 6{ }^{+} \mathrm{CCR} 4^{+}$cells, $\mathrm{CCR} 6^{-} \mathrm{CCR} 4^{+}$cells, and $\mathrm{CCR} 6^{+} \mathrm{CCR} 4^{+}$cells (Figure 3A) . Evaluation of $\mathrm{T}$ cell subset master regulators indicated that ROR $\gamma t$ was exclusively highly expressed in the $\mathrm{CCR} 6^{+} \mathrm{CCR} 4^{+}$subpopulation while GATA3 was predominantly expressed in the $\mathrm{CCR} 6^{-} \mathrm{CCR} 4^{+}$subpopulation(Figure 3B) . Consistently, analysis of IL-17A expression confirmed that the $\mathrm{CCR} 6^{+} \mathrm{CCR} 4^{+}$subpopulation was Th17 cells (Figure 3C \& 3D) . Previous studies have reported the significance of IL-17RE and CD27 to Th17 activity $[14,15]$. Therefore, we further dissected the CCR6 ${ }^{+} \mathrm{CCR} 4^{+}$subpopulation based on the expression of IL-17RE and CD27. As shown in Figure 3E , the $\mathrm{CCR} 6^{+} \mathrm{CCR} 4^{+}$subpopulation was divided into three subsets: IL-17RE ${ }^{+} \mathrm{CD} 27^{-}$cells, IL-17RE ${ }^{+} \mathrm{CD} 27^{+}$cells, and IL-17RE ${ }^{-} \mathrm{CD} 27^{-}$cells. The IL-17RE ${ }^{+} \mathrm{CD} 27^{-}$subset and IL-17RE ${ }^{+} \mathrm{CD} 27^{+}$subset expressed higher IL-17A than the IL-17RE-CD27-subset (Figure 3F). However, their ROR ${ }^{-}$t expression was comparable (Figure 3G) . Therefore, IL-17RE and CD27 signify the heterogeneity of PF Th17 cells.

Assessment of the expression of other Th17-related cytokines indicated that in comparison with the IL17RE ${ }^{-} \mathrm{CD} 27^{-}$subset, the IL-17RE ${ }^{+} \mathrm{CD} 27^{-}$subset and IL-17RE ${ }^{+} \mathrm{CD} 27^{+}$subset produced more GM-CSF (Figure 4A \& 4B) as well as IL-22 mRNA (Figure 4C) . Furthermore, evaluation of cell cycle using Hoechst 33342 and Pyronin Y demonstrated that the IL-17RE ${ }^{+}$CD27 $7^{-}$subset and IL-17RE ${ }^{+}$CD27 $7^{+}$subset had more cells in G1 phase and S-G2/M phase than the IL-17RE ${ }^{-} \mathrm{CD} 27^{-}$subset, suggesting that the former two subsets were more proliferative than the latter(Figure 4D). Therefore, IL-17RE ${ }^{+}$Th17 cells were more pro-inflammatory than IL-17RE- Th17 cells. CD27 seemed not profoundly influencing Th17 activity because no significant difference was seen between the IL-17RE ${ }^{+} \mathrm{CD} 27^{-}$subset and IL-17RE ${ }^{+} \mathrm{CD} 27^{+}$subset.

To deeply understand the molecular traits of PF IL-17RE ${ }^{+}$Th17 cells and IL-17RE-Th17 cells, we sorted these cells to conduct the RNA-Seq analysis(Figure 5A) . Compared with IL-17RE- Th17 cells, a total of 5288 differentially expressed transcripts (DETs) were identified in IL-17RE ${ }^{+}$Th17 cells, including 3866 up-regulated DETs and 1422 down-regulated DETs (Figure 5B \& 5C). The GO biological process (BP) enrichment results showed significant associations with respiratory electron transport chain, oxidative phosphorylation, and ATP synthesis coupled transport, etc.(Figure 5D) . GO cellular component (CC) analysis showed notable associations with respiratory chain, ribosome, and ribosomal subunit, etc. (Figure 5E) . GO molecular function (MF) analysis showed remarkable associations with structural molecular activity, structural constituent of ribosome, RNA binding, NADH dehydrogenase (ubiquinone) activity, and other metabolism-related activity (Figure 5F). KEGG analysis also enriched DETs in certain KEGG terms such as ribosome, oxidative phosphorylation, olfactory transduction, and glycolysis/gluconeogenesis (Figure 5G) . Hence, the expression of genes for OXPHOS and ETC seemed to be remarkably increased in IL-17RE ${ }^{+}$ Th17 cells relative to IL-17RE- Th17 cells.

Analysis of metabolism-related DETs revealed that in comparison to PF IL-17RE- Th17 cells, PF IL$17 \mathrm{RE}^{+}$Th17 cells up-regulated the expression of genes involved in OXPHOS and ETC, including NDUFB5 ,MT-CO1, UQCRC1, andNDUFS6, etc. (Figure 6A and Table.1). The up-regulation of these genes was validated by qRT-PCR (Figure 6B). Since OXPHOS and ETC are crucial for the generation of ATP and ROS, we quantified ATP and ROS in the two Th17 subsets. As shown in Figure 6C, IL-17RE ${ }^{+}$Th17 cells produced more ATP than IL-17RE- Th17 cells. H2DCFDA staining suggested more ROS generation in IL-17RE ${ }^{+}$Th17 cells relative to IL-17RE- Th17 cells (Figure 6D) . Therefore, IL-17RE ${ }^{+}$Th17 cells and IL-17RE-Th17 cells are metabolically different.

\section{Discussion}

In the current study, we developed a method to sort live Th17 cells from PF of severe endometriosis patients to evaluate the function of pathological Th17 cells. We are the first to use CXCR3, CCR4, and CCR6 isolate PF Th17 cells from endometriosis patients. In the future, it would be necessary to detect more surface markers to precisely pinpoint Th17 cells. Furthermore, detection of the above chemokine receptors would recognize Th1, Th2, and Th1/Th17 subsets and thus greatly facilitates research on T cell biology at endometriosis lesions. 
Th17 cells are heterogeneous and possess a remarkable degree of plasticity [16]. Our study indicates that PF Th17 cells are heterogeneous according to the expression of CD27 and IL-17RE. CD27 is a member of TNF receptor family expressed on $\mathrm{CD} 4^{+} \mathrm{T}$ cells at the priming or effector stage, while its ligand, CD70, is expressed on activated dendritic cells and lymphocytes [17]. Recent research suggests its inhibitory effect on Th17 cells through repressing transcription of IL-17 and CCR6 [15]. IL-17RE forms a receptor complex with IL-17RA to bind to IL-17C to potentiate the Th17 cell response [14]. IL-17C is preferentially secreted by epithelial cells in the lung, skin, and colon [18]. Interestingly, the Human Protein Atlas describes high IL-17C expression in the endometrium (https://www.proteinatlas.org/ENSG00000124391-IL17C/tissue), strongly suggesting the existence of IL-17C in endometriosis lesions. Our ongoing research is investigating the levels of IL-17C in PF and endometriosis tissues. Besides, our data indicate that IL-17RE rather than CD27 marks a more active Th17 subpopulation, because IL-17RE ${ }^{+}$Th17 cells expressed more Th17-related cytokines and were more proliferative than IL-17RE-Th17 cells. A possible explanation is that IL-17RE-triggered signaling overcomes CD27-induced signaling.

Through RNA-Seq, we found that most of the top 20 up-regulated metabolic genes in IL-17RE ${ }^{+}$Th17 cells encode enzymes involved in OXPHOS and ETC, while most of the down-regulated metabolic genes were associated with other metabolic pathways. It has been known that mitochondrial respiration is enhanced during Th17 differentiation and OXPHOS is essential for Th17 cell pathogenic signature gene expression [19]. Besides, Th17 cells have limited capacity to promote glycolysis to react to metabolic stresses and they rely on OXPHOS for the production of energy and cytokines [20]. Indeed, we observed higher proinflammatory activity associated with higher ATP and ROS in IL-17RE ${ }^{+}$Th17 cells. This suggests that OXPHOS and ETC support the pathogenicity of PF IL-17RE ${ }^{+}$Th17 cells in endometriosis. Our data also poses an interesting question: whether the IL-17C/IL-17RE axis results in the up-regulation of OXPHOS and ETC-related genes. This could be answered in future studies.

What remains unknown is the origin of the heterogeneous PF Th17 subsets. Are they a CD4 ${ }^{+} \mathrm{T}$ cell population at different maturation statuses? Or do they arise from different $\mathrm{CD} 4^{+} \mathrm{T}$ cell subsets under distinct microenvironment factors? Lineage tracing of Th17 cells in animal models and single-cell transcriptome sequencing might give the answer.

In conclusion, this study provides a novel method to detect and isolate live PF Th17 cells from endometriosis patients and unveils the functional and metabolic heterogeneity of PF Th17 subsets. Furthermore, the data of other differentially expressed genes shed light on the elucidation of other molecular mechanisms that modulate the function of pathological Th17 cells in endometriosis.

\section{References}

1 Chapron, C., Marcellin, L., Borghese, B. and Santulli, P., Rethinking mechanisms, diagnosis and management of endometriosis. Nat Rev Endocrinol 2019. 15: 666-682.

2 Vallve-Juanico, J., Houshdaran, S. and Giudice, L. C., The endometrial immune environment of women with endometriosis. Hum Reprod Update 2019. 25: 564-591.

3 Izumi, G., Koga, K., Takamura, M., Makabe, T., Satake, E., Takeuchi, A., Taguchi, A., Urata, Y., Fujii, T. and Osuga, Y., Involvement of immune cells in the pathogenesis of endometriosis. J Obstet Gynaecol Res 2018. 44: 191-198.

4 Szyllo, K., Tchorzewski, H., Banasik, M., Glowacka, E., Lewkowicz, P. and Kamer-Bartosinska, A., The involvement of $\mathrm{T}$ lymphocytes in the pathogenesis of endometriotic tissues overgrowth in women with endometriosis. Mediators Inflamm2003. 12: 131-138.

5 Tanaka, Y., Mori, T., Ito, F., Koshiba, A., Takaoka, O., Kataoka, H., Maeda, E., Okimura, H., Mori, T. and Kitawaki, J., Exacerbation of Endometriosis Due To Regulatory T-Cell Dysfunction. J Clin Endocrinol Metab 2017. 102: 3206-3217.

6 Takamura, M., Koga, K., Izumi, G., Hirata, T., Harada, M., Hirota, Y., Hiraike, O., Fujii, T. and Osuga, Y., 
Simultaneous Detection and Evaluation of Four Subsets of CD4+ T Lymphocyte in Lesions and Peripheral Blood in Endometriosis. Am J Reprod Immunol 2015. 74: 480-486.

7 Gogacz, M., Winkler, I., Bojarska-Junak, A., Tabarkiewicz, J., Semczuk, A., Rechberger, T. and Adamiak, A., Increased percentage of Th17 cells in peritoneal fluid is associated with severity of endometriosis. $J$ Reprod Immunol 2016. 117: 39-44.

8 Zhao, F., Hoechst, B., Gamrekelashvili, J., Ormandy, L. A., Voigtlander, T., Wedemeyer, H., Ylaya, K., Wang, X. W., Hewitt, S. M., Manns, M. P., Korangy, F. and Greten, T. F., Human CCR4+ CCR6+ Th17 cells suppress autologous CD8+ T cell responses. J Immunol 2012. 188: 6055-6062.

9 Halim, L., Romano, M., McGregor, R., Correa, I., Pavlidis, P., Grageda, N., Hoong, S. J., Yuksel, M., Jassem, W., Hannen, R. F., Ong, M., McKinney, O., Hayee, B., Karagiannis, S. N., Powell, N., Lechler, R. I., Nova-Lamperti, E. and Lombardi, G., An Atlas of Human Regulatory T Helper-like Cells Reveals Features of Th2-like Tregs that Support a Tumorigenic Environment. Cell Rep 2017. 20: 757-770.

10 Pandya, J. M., Lundell, A. C., Hallstrom, M., Andersson, K., Nordstrom, I. and Rudin, A., Circulating $\mathrm{T}$ helper and $\mathrm{T}$ regulatory subsets in untreated early rheumatoid arthritis and healthy control subjects. $J$ Leukoc Biol 2016. 100: 823-833.

11 Gosselin, A., Monteiro, P., Chomont, N., Diaz-Griffero, F., Said, E. A., Fonseca, S., Wacleche, V., El-Far, M., Boulassel, M. R., Routy, J. P., Sekaly, R. P. and Ancuta, P., Peripheral blood CCR4+CCR6+ and CXCR3+CCR6+CD4+ T cells are highly permissive to HIV-1 infection. J Immunol 2010. 184: 1604-1616.

12 Mony, J. T., Khorooshi, R. and Owens, T., Chemokine receptor expression by inflammatory T cells in EAE.Front Cell Neurosci 2014. 8: 187.

13 Wang, C., Kang, S. G., Lee, J., Sun, Z. and Kim, C. H., The roles of CCR6 in migration of Th17 cells and regulation of effector T-cell balance in the gut. Mucosal Immunol2009. 2: 173-183.

14 Chang, S. H., Reynolds, J. M., Pappu, B. P., Chen, G., Martinez, G. J. and Dong, C., Interleukin-17C promotes Th17 cell responses and autoimmune disease via interleukin-17 receptor E. Immunity 2011. 35: 611-621.

15 Coquet, J. M., Middendorp, S., van der Horst, G., Kind, J., Veraar, E. A., Xiao, Y., Jacobs, H. and Borst, J., The CD27 and CD70 costimulatory pathway inhibits effector function of T helper 17 cells and attenuates associated autoimmunity.Immunity 2013. 38: 53-65.

16 Bystrom, J., Clanchy, F. I. L., Taher, T. E., Al-Bogami, M., Ong, V. H., Abraham, D. J., Williams, R. O. and Mageed, R. A., Functional and phenotypic heterogeneity of Th17 cells in health and disease. Eur $J$ Clin Invest 2019. 49: e13032.

17 Borst, J., Hendriks, J. and Xiao, Y., CD27 and CD70 in T cell and B cell activation. Curr Opin Immunol 2005. 17: 275-281.

18 Nies, J. F. and Panzer, U., IL-17C/IL-17RE: Emergence of a Unique Axis in TH17 Biology. Front Immunol 2020. 11: 341.

19 Shin, B., Benavides, G. A., Geng, J., Koralov, S. B., Hu, H., Darley-Usmar, V. M. and Harrington, L. E., Mitochondrial Oxidative Phosphorylation Regulates the Fate Decision between Pathogenic Th17 and Regulatory T Cells. Cell Rep 2020. 30: 1898-1909 e1894.

20 Franchi, L., Monteleone, I., Hao, L. Y., Spahr, M. A., Zhao, W., Liu, X., Demock, K., Kulkarni, A., Lesch, C. A., Sanchez, B., Carter, L., Marafini, I., Hu, X., Mashadova, O., Yuan, M., Asara, J. M., Singh, H., Lyssiotis, C. A., Monteleone, G., Opipari, A. W. and Glick, G. D., Inhibiting Oxidative Phosphorylation In Vivo Restrains Th17 Effector Responses and Ameliorates Murine Colitis.J Immunol 2017. 198: 2735-2746.

Figure captions 
Figure 1. The phenotype of PF IL-17-producing T cells in endometriosis. (A) Representative FLOW CYTOMETRY plots showing IL-17-producing T cells in endometriosis PF. Single cells were gated among the whole events, followed by gating lymphocytes based on FSC-A and SSC-A. CD3 ${ }^{+} \mathrm{IL}-17 \mathrm{~A}^{+}$cells were then observed in lymphocytes. Numbers in the quadrants indicate the percentages of cell populations. (B) Detection of indicated surface markers in PF IL-17-producing T cells. Isotype: isotype antibody control. Ab: specific antibody for corresponding markers. (C)Frequencies of $\mathrm{CCR} 6^{+} \mathrm{CCR} 4^{+}$and $\mathrm{CCR} 6^{+} \mathrm{CCR} 4^{-}$ subpopulations in IL-17-producing T cells. (D) Frequencies of IL-17RE ${ }^{+}$CD27-, IL-17RE ${ }^{+}$CD27 ${ }^{+}$, IL17RE $\mathrm{RD}^{-} 7^{+}$, and $\mathrm{IL}-17 \mathrm{RE}^{-} \mathrm{CD} 27^{-}$subset in IL-17-producing $\mathrm{T}$ cells. $\mathrm{N}=5$ individuals per group.
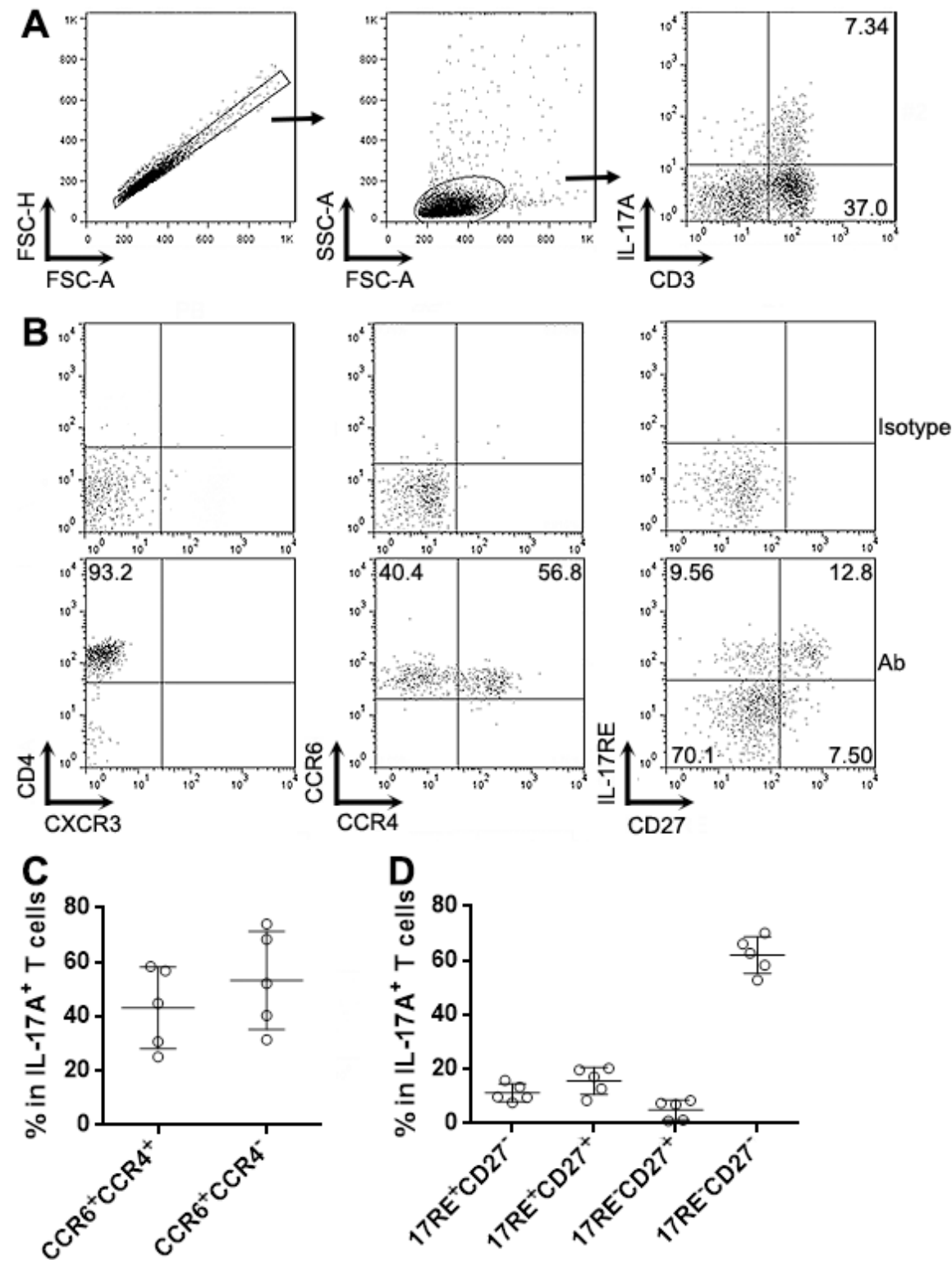

Figure 2. Identification of live PF Th17 cells according to CCR6 and CCR4. (A) Gating of $\mathrm{CD}^{+}{ }^{+} \mathrm{CD} 4^{+} \mathrm{T}$ cells in endometriosis PF and normal blood. PF: peritoneal fluid. PB: peripheral blood. (B) $\mathrm{T}$ cell subsets based on the expression of CCR4 and CXCR3. PB: blood CD3 ${ }^{+} \mathrm{CD} 4^{+} \mathrm{T}$ cells as control. (C) Relative mRNA levels of indicated master regulators in CCR4-and-CXCR3-defined $\mathrm{T}$ cell subsets. 
(D)Subpopulations in CCR $4^{+} \mathrm{CXCR}^{-} \mathrm{T}$ cells based on the expression of CCR6 and CCR10. (E) Relative mRNA levels of indicated master regulators in the subpopulations described in (D). ${ }^{*}: p<0.05 ;{ }^{* *}: p<0.01$; $* * *: p<0.001$. $\mathrm{N}=6$ individuals per group. Kruskal-Wallis test.
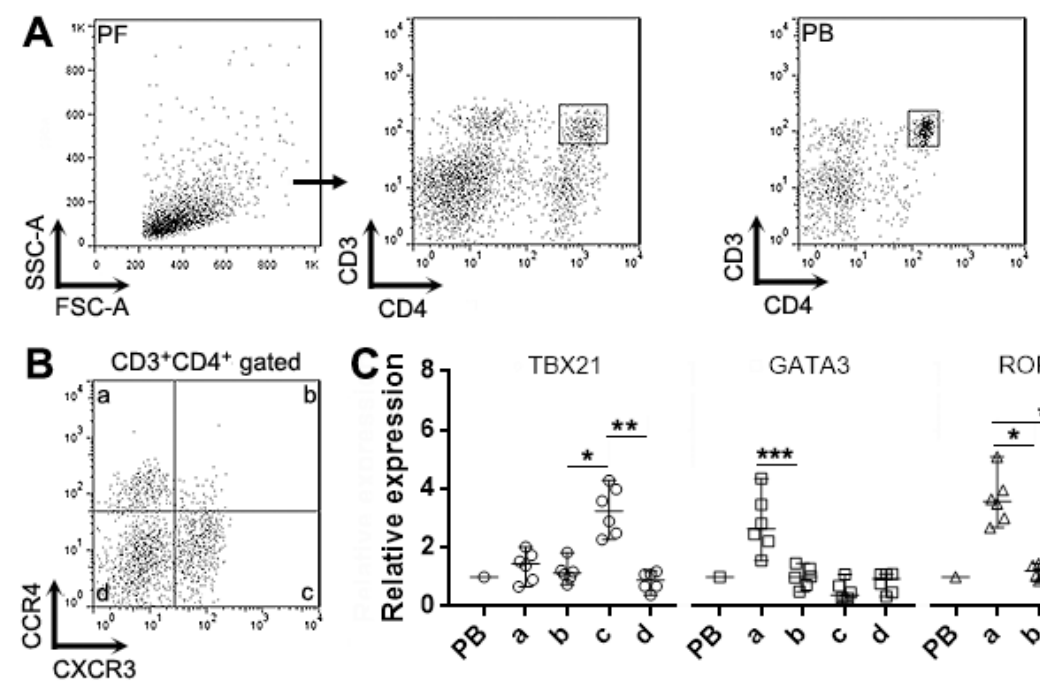

GATA3

ROR $\gamma \mathrm{t}$
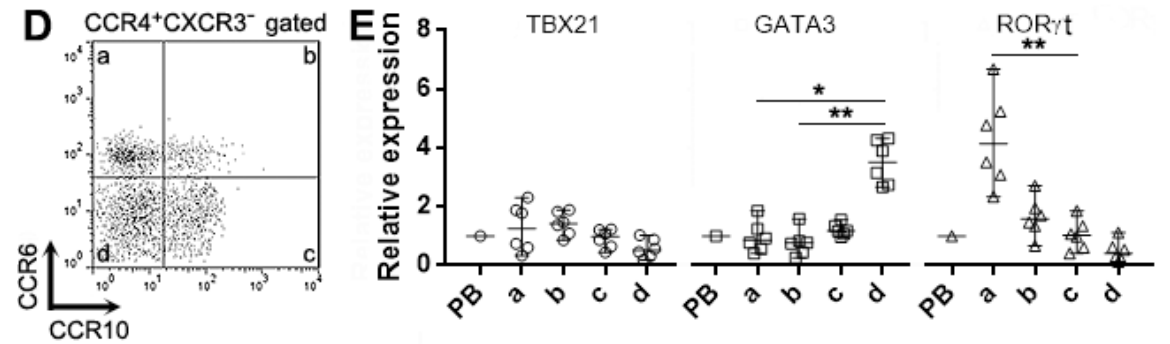

Figure 3. Identification of live PF Th17 subsets according to IL-17RE and CD27. (A) Subsets in CD14-CXCR3-CCR10-CD4+ cells based on the expression of CCR6 and CCR4. (B) Relative mRNA levels of indicated master regulators in the subpopulations described in (A). PB: blood CD3+CD4+ T cells as control. R6: CCR6. R4: CCR4. N=3 per group. (C) Flow cytometry plots showing IL-17A expression in the subpopulations described in (A). (D) Frequencies of IL-17A+ cells in the 4 subpopulations. (E) Expression of IL-17RE and CD27 on the surface of the CCR6+CCR4+ subpopulation. (F) Relative mRNA levels of IL-17A in IL-17RE+CD27- cells, IL-17RE+CD27+ cells, and IL-17RE-CD27- cells. (G) Relative mRNA levels of ROR $\gamma t$ in the same three subsets as (F). ). *: $\mathrm{p}<0.05$; $^{* *}: \mathrm{p}<0.01{ }^{* * *}: \mathrm{p}<0.001$. $\mathrm{N}=5$ per group in (D) to $(\mathrm{G})$. Kruskal-Wallis test. 

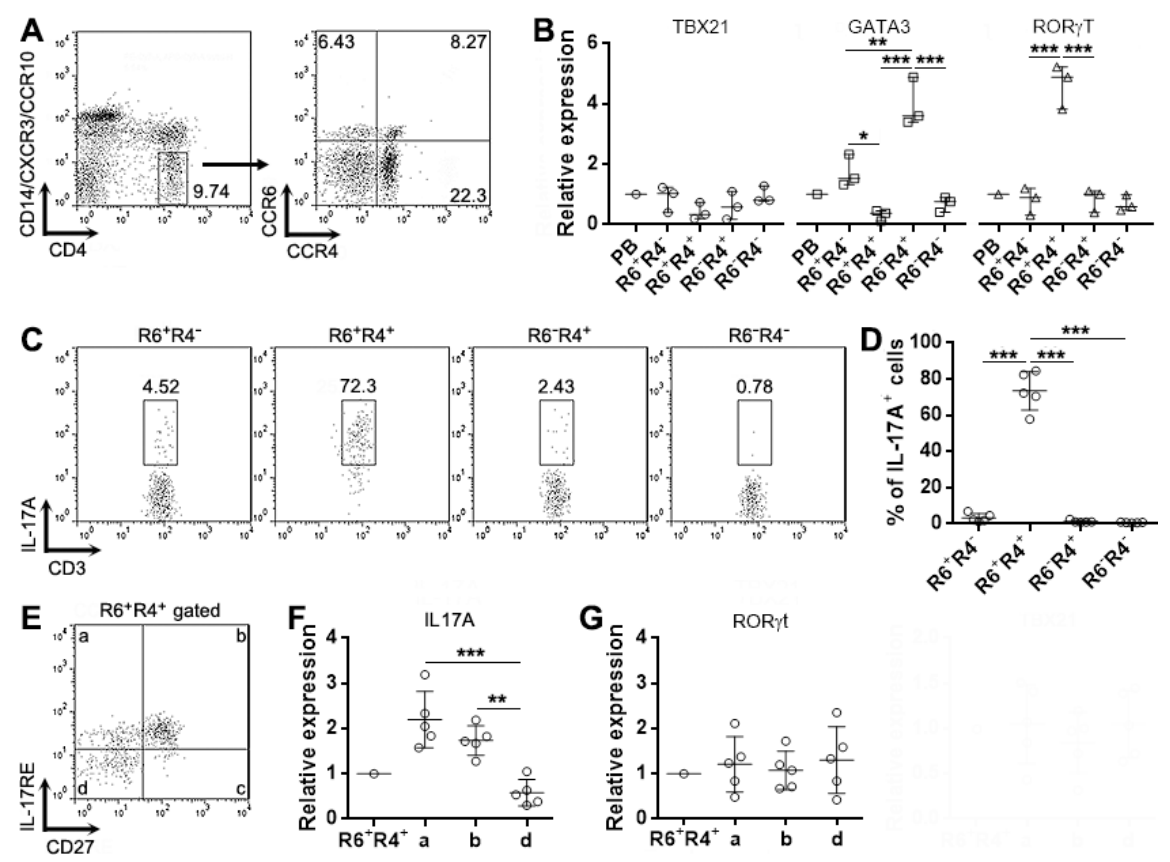

Figure 4. Functions of PF Th17 subsets. (A \& B) GM-CSF expression in IL-17RE ${ }^{+}$CD27 $^{-}$subset, IL-17RE ${ }^{+} \mathrm{CD}_{27}{ }^{+}$subset, and IL-17RE-CD27- ${ }^{-}$subset. Representative flow cytometry plots are shown in (A), and statistics of the frequencies of $\mathrm{GM}_{-} \mathrm{CSF}^{+}$cells are shown in (B). (C)Relative mRNA levels of IL22 in the three subsets. $\mathrm{N}=5$ per group. Kruskal-Wallis test. (D) Flow cytometry plots showing cell cycle measured by staining with Hoechst 33342 and Pyronin Y. ${ }^{*}: p<0.05 ;{ }^{*}: p<0.01$. 

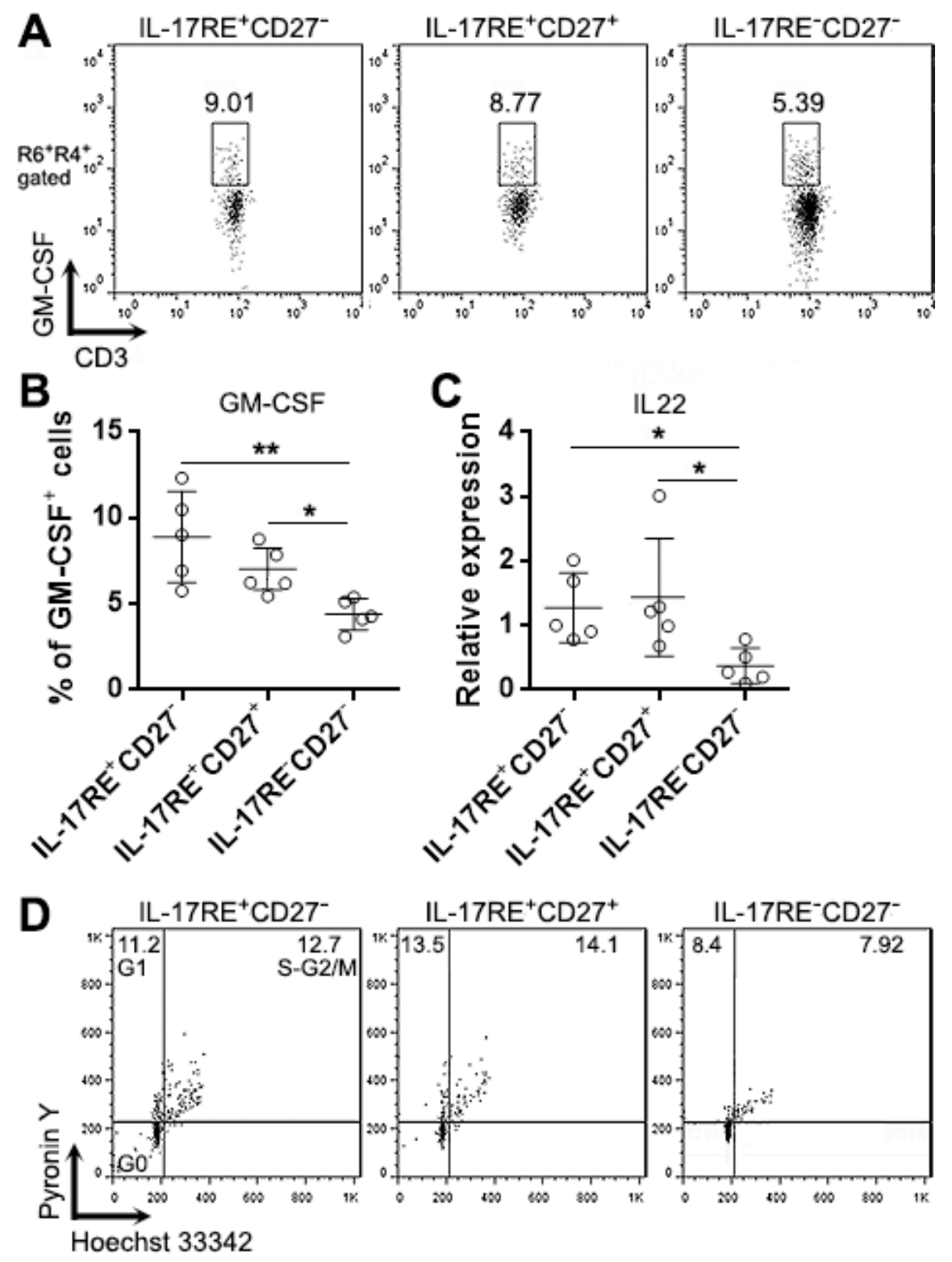

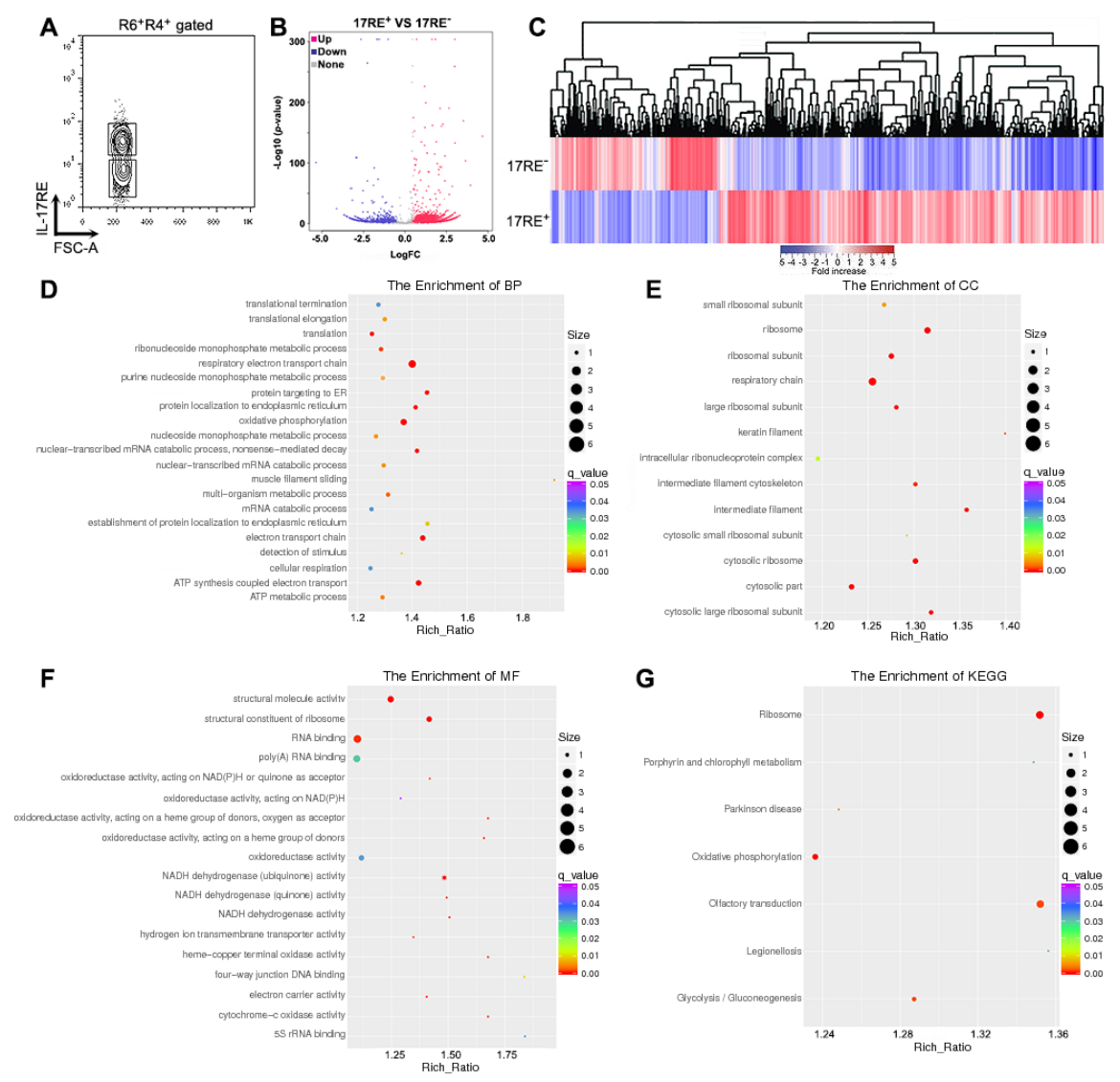

Figure 5. RNA-Seq analysis of PF IL-17RE+ Th17 cells and IL-17RE- Th17 cells. (A) Gating and sorting of PF CCR6+CCR4+IL-17RE+ Th17 cells and CCR6+CCR4+IL-17RE- Th17 cells. (B) Volcano plot displaying the DETs. (C) Cluster analysis of DETs. 17RE-: IL-17RE- Th17. 17RE+: IL-17RE+ Th17. (D to F) Enrichment of up-regulated DETs for GO terms in the category of biological process terms (D), cellular component terms (E), and molecular function terms (F). (G) Enrichment of up-regulated DETs for KEGG terms.

Figure 6. OXPHOS and ETC in PF IL-17RE+ Th17 cells and IL-17RE- Th17 cells. (A) Heat map of top 40 metabolism-related DETs (FDR < 0.05). (B) Validation of DETs in (A) by qRT-PCR. $\mathrm{N}=5^{\sim} 7$ per group. The data are presented as min to max. (C) ATP levels in IL-17RE ${ }^{+}$Th17 cells and IL-17RE-Th17 cells. $\mathrm{N}=6$ per group. (D) ROS levels measured by H2DCFDA staining. $\mathrm{N}=5$ per group. *: $\mathrm{p}<0.05 ;^{* *}: \mathrm{p}<0.01 ;^{* * *}: \mathrm{p}<0.001$. Mann-Whitney U test. 

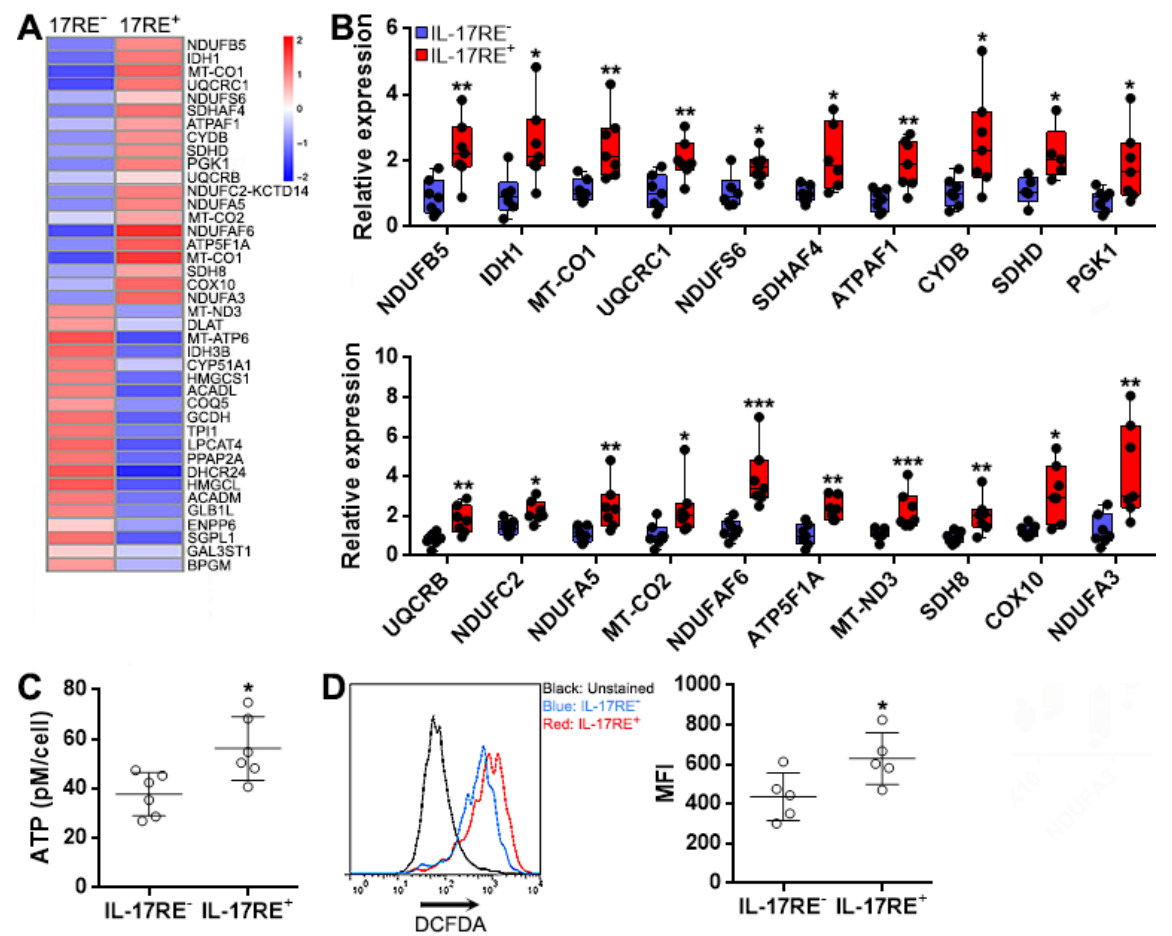

All data included in this study are available upon request by contact with the corresponding author.

Conflicts of interest

The authors declare no conflict of interest with regard to the publication of this paper.

There are no funders to report for this submission.

Author contributions:

Yanping Jiang performed experiment and the data analyses, and wrote the manuscript;

Lu Wang performed the experiment and wrote the manuscript;

Yanping Jiang and Lu Wang contributed equally to this work and should be considered co-first authors.

Yaqin Peng contributed significantly to analysis and manuscript preparation;

Jian Qin contributed to the conception of the study

Aili Tan helped perform the analysis with constructive discussions.

Shujun Wang helped perform data analyses 